\title{
Estudo de materiais naturais para aplicação em isolamento térmico de edificações
}

As crises energéticas e os impactos climáticos revelaram a necessidade de se repensar a forma de projetar as edificações, que se apresentam como grande geradores de gases causadores do efeito estufa e consumo de $40 \%$ da energia elétrica mundial. A ONU e diversos países vem redigindo recomendações e legislações sobre o tema, orientando a utilização de materiais adequados, visando a eficiência energética em edificações. Este estudo se desenvolveu a partir de uma metodologia de pesquisa bibliométrica, buscando por materiais de isolamento térmico para utilização em edificações, que possam substituir materiais convencionais que utilizam recursos não renováveis e poluentes no seu sistema de fabricação. Grande parte dos materiais naturais pesquisados, surgem oriundo de resíduos agrícolas, que podem ser tratados, e utilizados como material construtivo. Os estudos pesquisados apontam desempenho de condutividade térmica ( $\lambda$ ) abaixo do recomendado para materiais isolantes $(\lambda<0,07 \mathrm{~W} / \mathrm{m} . \mathrm{K})$. Assim, materiais que poderiam ser considerados resíduos, surgem como nova alternativa construtiva. Os estudos desenvolvidos a partir da casca de arroz $(\lambda=0,0464 \mathrm{~W} / \mathrm{m} . \mathrm{K})$, folha de abacaxi $(\lambda=0,0380 \mathrm{~W} / \mathrm{m} . \mathrm{K})$, bagaço de cana $(\lambda=0,0460 \mathrm{~W} / \mathrm{m} . \mathrm{K})$ e sabugo de milho $(\lambda=0,0580 \mathrm{~W} / \mathrm{m} . \mathrm{K})$ apresentam resultados importantes, além de serem alternativas viáveis para serem utilizadas no Brasil, por haver grandes áreas de plantio para estas culturas. Salienta-se a importância de se desenvolver pesquisas práticas da aplicabilidade destes materiais, bem como testes de resistência ao fogo e Avaliação do Ciclo de Vida (ACV).

Palavras-chave: Condutividade Térmica; Isolamento Térmico; Conforto Térmico; Eficiência Energética; Material Natural.

\section{Study of natural materials for application in thermal insulation of buildings}

\begin{abstract}
Energy crises and climate impacts have revealed the need to rethink the design of buildings, which are major generators of greenhouse gases and $40 \%$ of the world's electricity consumption. The UN and several countries have been writing recommendations and legislation on the subject, guiding the use of appropriate materials, aiming at energy efficiency in buildings. This study was developed from a bibliometric research methodology, looking for thermal insulation materials for use in buildings that can replace conventional materials that use non-renewable resources and pollutants in their manufacturing system. Most of the natura materials researched come from agricultural residues, which can be treated and used as constructive material. The studies researched show thermal conductivity performance $(\lambda)$ below the recommended for insulating materials $(\lambda<0.07 \mathrm{~W} / \mathrm{m} . \mathrm{K})$. Thus, materials that could be considered waste appear as a new constructive alternative. Studies from rice husk $(\lambda=0.0464 \mathrm{~W} / \mathrm{mK})$, pineapple leaf $(\lambda=0.0380 \mathrm{~W} / \mathrm{mK})$, sugarcane bagasse $(\lambda=0.0460 \mathrm{~W} / \mathrm{mK})$ and Corn $(\lambda=0.0580 \mathrm{~W} / \mathrm{mK}) \mathrm{has}$ important results, besides being viable alternatives to be used in Brazil, because there are large areas of planting for these crops. The importance of developing practical research on the applicability of these materials, as well as fire resistance testing and Life Cycle Assessment (LCA) is emphasized.
\end{abstract}

Keywords: Thermal Conductivity; Thermal Insulation; Thermal Comfort; Energy Efficiency; Natural Material.

Topic: Construções e Arquitetura Sustentáveis

Reviewed anonymously in the process of blind peer.
Received: 10/02/2018

Approved: $24 / 03 / 2018$
Rodrigo Spinelli (iD

Universidade do Vale do Taquari, Brasil

http://lattes.cnpq.br/7771857527009401

http://orcid.org/0000-0002-6323-706X

rspinelli@univates.br

Faustino Patiño Cambeiro (iD

Universidade de Vigo, Espanha

http://lattes.cnpq.br/3732275572091623

http://orcid.org/0000-0002-2340-7861

faustinopc@gmail.com

Odorico Konrad

Universidade do Vale do Taquari, Brasil

http://lattes.cnpq.br/9946679953072196

http://orcid.org/0000-0002-6968-7969

okonrad@univates.br
Referencing this:

SPINELLI, R.; CAMBEIO, F. P.; KONRAD, O.. Estudo de materiais naturais para aplicação em isolamento térmico de edificações. Revista Ibero Americana de Ciências Ambientais, v.9, n.3, p.121-135, 2018. DOI: http://doi.org/10.6008/CBPC2179-6858.2018.003.0011 


\section{INTRODUÇÃO}

A crise energética desencadeada no início dos anos 1970, apresenta uma realidade em que se torna fundamental trabalhar com estratégias que visam minimizar o consumo energético, e a racionalização dos métodos de utilização dos recursos naturais (SPINELLI et al., 2013). Para Muniz et al. (2015), intensificam-se os alertas climáticos

com a criação e atuação do clube de Roma e a conferência de Estocolmo na Suécia em 1972 onde, dentre outros temas ambientais, apresentou-se o conceito de desenvolvimento sustentável, defendendo o uso moderado dos recursos naturais, tanto por serem finitos como por haver preocupação no que tange às fragilidades de alguns ambientes naturais.

Em novembro de 2016, entra em vigor do Acordo de Paris, visa reduzir consideravelmente as emissões de gases causadores do efeito estufa, a fim de reduzir a ampliação da temperatura média do planeta, que se encaminha para níveis alarmantes, pois "2015 foi o ano mais quente do mundo moderno, e os seis primeiros meses de 2016, até agora, bateram todos os recordes anteriores".

Os impactos das construções ao meio ambiente para a união europeia, em relação as emissões de $\mathrm{CO}_{2}$, as edificações estão entre os consumidores de energia mais significativos e os emissores de gases de efeito estufa; habitações, escritórios, lojas e o resto de edifícios públicos e privados geram $36 \%$ das emissões de gás e representam $40 \%$ do consumo de energia (EICHHAMMER et al., 2009). Destaca-se que o mercado da construção civil utiliza aproximadamente $40 \%$ dos recursos naturais não renováveis do planeta (JOURDA, 2012). Os dados apresentados pelo Programa das Nações Unidas para o Meio Ambiente (UNEP, 2000, citado por ASDRUBALI et al., 2015) validam estes percentuais, e reforçam que $25 \%$ da água global é utilizada pelas edificações.

A estratégia desenvolvida pela União Europeia a fim de mitigar os efeitos negativos da mudança climática, em que até o ano de 2020 as edificações novas deverão ser eficientes energeticamente, com balanço próximo do zero, consumindo a mesma quantidade de energia que seja capaz de gerar, cabendo a cada estado membro desenvolver o melhor método para atingir os objetivos, e apresenta-se como fator fundamental a adaptação climática das edificações, seguindo as orientações da Diretiva 2010/31/EU do Parlamento Europeu, Relativa ao Desempenho Energético dos Edifícios, determinando que os materiais de construção sejam utilizados de forma racional, as edificações devem ser eficientes energeticamente para minimizar a emissão dos gases de efeito estufa (CAMBEIRO et al., 2016).

No Brasil, segundo dados da Empresa de Pesquisa Energética (EPE, 2017), os setores Residencial (29\%), Comercial (19\%), e Industrial (36\%), são responsáveis por $84 \%$ do consumo energético atual, considerado elevado (PROCEL INFO, 2017). Em relatório elaborado pela EPE (2016), em que apresenta "O Compromisso do Brasil no Combate às Mudanças Climáticas: Produção e Uso de Energia", identifica que para o consumo energético há três desafios importantes a serem atendidos: 1) eficiência energética para redução do consumo energético de edificações e emissões de Gases de Efeito Estufa (GEE); 2) ampliação da autoprodução energética, para que atenda o crescente consumo e da geração distribuída; e 3) atender um crescimento de consumo energético anual de 3\%, no período de 2014 a 2030 (desconsiderando a autoprodução e incluindo os ganhos relacionados a eficiência energética) (EPE, 2016). 
Destaca-se no relatório desenvolvido pela EPE (2016) o papel do Programa Nacional de Conservação de Energia Elétrica (PROCEL) ${ }^{1}$, que por meio do PROCEL Edifica busca estimular a utilização dos conceitos de eficiência energética em edificações, apoiando a viabilização da Lei de Eficiência Energética (10.295/2001), "bem como contribuir com a expansão, de forma energeticamente eficiente, do setor de edificações do país, reduzindo os custos operacionais na construção, utilização e manutenção dos imóveis" (PROCEL, 2016).

Ao se projetar de forma eficiente as edificações, segundo dados do PROCEL INFO (2017), edificação novas, que tendem a utilizar estratégias de eficiência energética, apresentam uma economia de até $50 \%$, e as edificações reformadas podem apresentar uma economia de até 30\%. Em edificações públicas, cerca de $70 \%$ da energia é utilizada em iluminação e climatização artificial, o que reforça a importância do tratamento adequado de fechamentos em paredes e aberturas, com a utilização de materiais com isolamento térmico (PROCEL INFO, 2017).

Em estudo a partir da análise de dados climáticos, e desenvolvimento da carta bioclimática (Figura 1) para a cidade de Lajeado/RS, descreve não haver necessidade de utilização de estratégias de climatização artificial, tanto para resfriamento e aquecimento (zonas 5 e 9), retratando assim a inadequação dos materiais construtivos atualmente utilizados para o clima local. Reforça assim a importância de utilização de materiais inovadores para isolamento térmico e eficiência energética de edificações (SPINELLI et al., 2017).

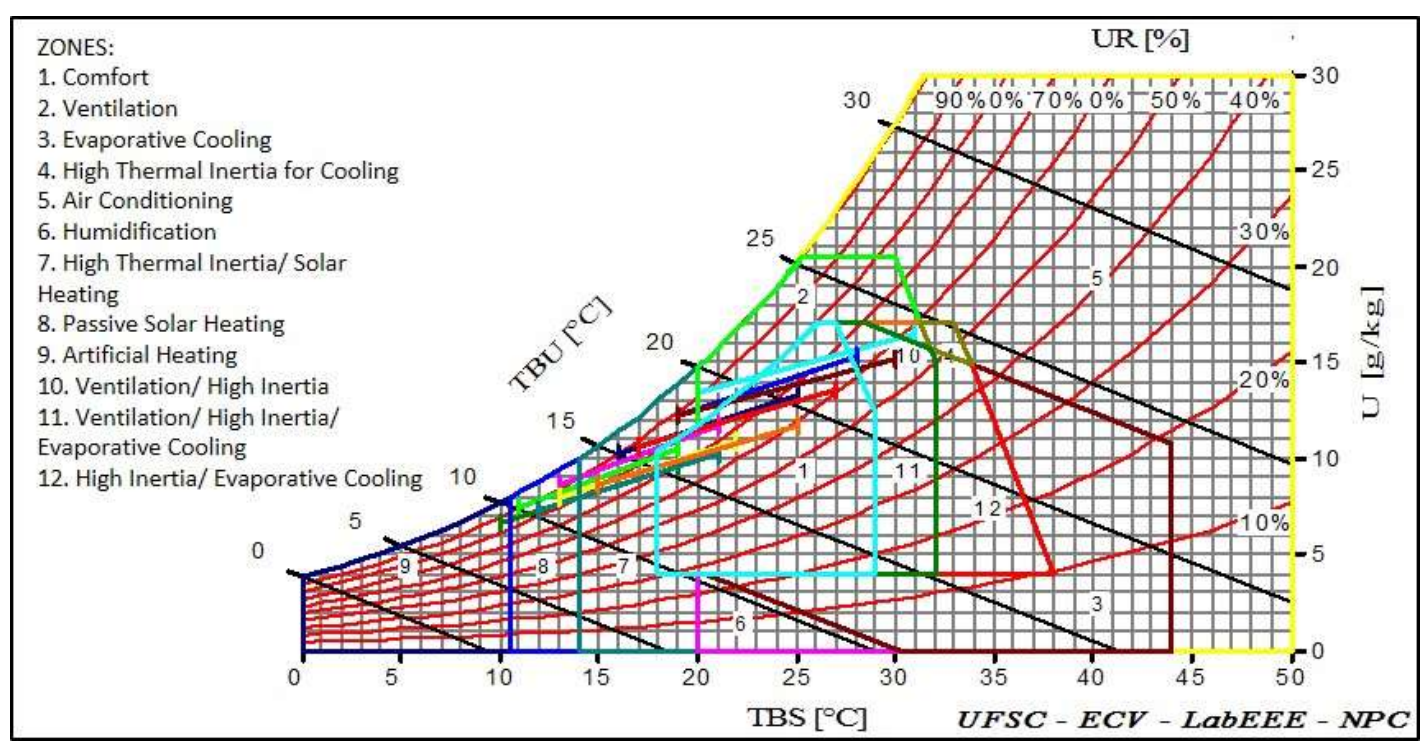

Figura 1: Carta Bioclimática para Lajeado/RS. Fonte: Spinelli et al. (2017).

OBS.: Zona 1 - Conforto Térmico; Zona 2 - Ventilação Natural; Zona 3 - Resfriamento Evaporativo; Zona 4 - Alta Inércia Térmica para Resfriamento; Zona 5 - Ar Condicionado; Zona 6 - Umidificação; Zona 7 - Alta Inércia / Aquecimento Solar Passivo; Zona 8 - Aquecimento Solar Passivo; Zona 9 - Aquecimento Artificial; Zona 10 Ventilação Natural / Alta Inércia; Zona 11 - Ventilação Natural / Alta Inércia / Resfriamento Evaporativo; Zona 12 - Alta Inércia / Resfriamento Evaporativo.

\section{REVISÃO TÉORICA}

\section{Materiais Convencionais Utilizados para Isolamento Térmico}

Os materiais comercialmente utilizados para isolamento térmico são: 1) Lã de rocha (ou lã mineral), produzido à base de rocha liquefeita, e com baixa condutividade térmica $(\lambda=0,035 / 0,040 \mathrm{~W} / \mathrm{m} . \mathrm{K}) ; 2)$ Lã de

\footnotetext{
1 É um programa de governo, coordenado pelo Ministério de Minas e Energia - MME e executado pela Eletrobrás. Foi instituído em 30 de dezembro de 1985 para promover o uso eficiente da energia elétrica e combater o seu desperdício. As ações do Procel contribuem para o aumento da eficiência dos bens e serviços, para o desenvolvimento de hábitos e conhecimentos sobre o consumo eficiente da energia e, além disso, postergam os investimentos no setor elétrico, mitigando, assim, os impactos ambientais e colaborando para um Brasil mais sustentável (PROCEL, 2016).
} 
vidro, produzida pela expansão do vidro a quente, e condutividade térmica baixa $(\lambda=0,04 / 0,055 \mathrm{~W} / \mathrm{m} . \mathrm{K})$; 3) Poliuretano (PU), que apresenta características adequadas para utilização na construção civil, e condutividade térmica muito baixa $(\lambda=0,025 / 0,040 \mathrm{~W} / \mathrm{m} . \mathrm{K}) ; 4)$ Poliuretano Projetado $(\lambda=0,016 / 0,02$ $\mathrm{W} / \mathrm{m} . \mathrm{K}) ; 5$ ) Poliuretano Expandido (EPS), um dos materiais mais utilizados para isolamento térmico ( $\lambda=0,035$ /0,040 W/m.K); e 6) Poliuretano Extrudado (XPS) ( $\lambda=0,035$ /0,040 W/m.K) (DUTRA, 2010).

Um material pode ser considerado como um isolador térmico, se o seu índice de condutividade térmica for inferior a 0,07 W/m.K. Wiebeck et al. (2005) apresenta dados relacionados aos polímeros sintéticos (EPS e XPS), como sendo materiais com boa resistência ao fogo, isolantes acústicos, e não propagam fungos. Em sua composição, os polímeros (PU) possuem isocianatos ${ }^{2}$, sendo substâncias voláteis, oferecem risco a saúde das pessoas que os inalarem (ASDRUBALI et al., 2005a). Marques et al. (2009) citam que:

Os polímeros sintéticos, que foram responsáveis por muitos avanços que temos hoje como a obtenção de materiais plásticos, e a infinidade de itens que constituem o nosso cotidiano, também são responsáveis por grandes problemas com o meio ambiente. Muitos polímeros não são recicláveis e os sintéticos derivados de petróleo não sofrem biodegradação, e ainda os que são recicláveis muitas vezes não são reciclados, causando problemas imensuráveis como enchentes, que por sua vez causam problemas ambientais e sociais, nos colocando assim em uma bola de neve catastrófica.

\section{Normatização}

Para a normatização do desempenho térmico de materiais, a Associação Brasileira de Normas Técnicas (ABNT) elaborou a NBR 15220, que apresenta os índices a serem necessários para se calcular o Desempenho Térmico de Edificações. O índice fundamental, é a condutividade térmica $(\lambda)$, que segundo a NBR 15220-1 é a "Propriedade física de um material homogêneo e isótropo, no qual se verifica um fluxo de calor constante, com densidade de $1 \mathrm{~W} / \mathrm{m}^{2}$, quando submetido a um gradiente de temperatura uniforme de 1 Kelvin por metro", e sua unidade de medida é W/(m.K) (ABNT, 2005).

A NBR 15220-2, apresenta a condutividade térmica de diversos materiais convencionais. Na NBR 15220-4, detalha o método para determinação de resistência térmica e condutividade térmica com a utilização do princípio da placa quente protegida (ABNT, 2005). Com a determinação da Condutividade Térmica de um material, torna-se possível se calcular a Resistência Térmica $(R)^{3}$ de elementos e componentes, a Transmitância Térmica $(U)^{4}$, a Densidade do Fluxo de Calor $(q)^{5}$ de um fechamento opaco, e o Fluxo de Calor $(Q)^{6}$.

\footnotetext{
2 Em química, isocianato é o grupo funcional de átomos - $\mathrm{N}=\mathrm{C}=\mathrm{O}$ (nitrogênio, carbono e oxigênio). Um composto orgânico que tem este grupo funcional, tendo fórmula geral $R-N=C=O$ é chamado de um isocianato. O grupo Isocianato é altamente reativo a grupos com Hidrogênio livre no sistema, um exemplo típico são as tintas de Poliuretano bicomponente, no qual o isocianato reage com uma Hidroxilia OH, formando uma ligação uretânica. O isocianato é altamente higroscópico, devendo ser mantido longe de umidade, visto sua característica de reatividade com a água (McNaught, 2005).

3 "Quociente da diferença de temperatura verificada entre as superfícies de um elemento ou componente construtivo pela densidade de fluxo de calor, em regime estacionário" (ABNT, 2005).

4 "O inverso da resistência térmica total de um componente" (ABNT, 2005).

5 "Quociente do fluxo de calor que atravessa uma superfície pela área dessa superfície" (ABNT, 2005).

6 "Quociente da quantidade de calor que atravessa uma superfície durante um intervalo de tempo" (ABNT, 2005).
} 
Assim, tomada a importância do desempenho energético para a qualidade das edificações, elaborouse a NBR 15575, para se atender as exigências dos usuários de edificações habitacionais, comportamento este relacionado ao período do seu uso. A NBR 15575 é um conjunto de normas que estipula o desempenho de acordo com requisitos (qualitativos), critérios (quantitativos ou premissas) e métodos de avaliação que proporcionam a construção de edificações mais seguras e eficientes (ABNT, 2013).

A NBR 15575 destaca as exigências dos usuários das edificações residenciais, apresentando uma lista geral dividida em segurança, habitabilidade e sustentabilidade. Esta norma de desempenho complementa as normas pré-existentes, não substituíndo-las, em que as normas de desempenho e prescritivas devendo ser utilisadas em conjunto (ABNT, 2013). Asdrubali et al. (2015a) cita que "os sistemas e materiais de isolamento térmico visam reduzir a transmissão do fluxo de calor. O desempenho de isolamento térmico de materiais homogêneos únicos ou combinados é geralmente avaliado, respectivamente, por condutividade térmica e transmitância térmica".

\section{Certificações}

Destaca-se que as edificações são, consequentemente, uma das principais fontes de poluição em todo o mundo. Os modelos internacionais de certificação que atestam a sustentabilidade ambiental dos edifícios adotam o impacto ambiental da construção em seu ciclo de vida como característica fundamental. Atualmente, esses certificados atendem aos critérios locais (BREEAM, criado no Reino Unido, HQE na França, LIDERA em Portugal) ou têm um alcance universal, como é o caso certificação americana conhecida como LEED. Esses certificados levam em conta o desempenho energético do prédio como característica fundamental, baseadas no processo construtivo, materiais de construção e sua procedência de origem (sendo uma preocupação o transporte em longa distância, e quantidade de emissões de gases gerados, entre outras), bem como a energia utiliza para execução e manutenção da edificação (CAMBEIRO et al., 2016).

\section{Objetivo}

Esta pesquisa tem por objetivo apresentar um referencial bibliográfico de pesquisas relacionadas a materiais naturais, com potencial inovador para utilização em isolamento térmico de edificações. Para os materiais pesquisados, será desenvolvida uma descrição sintetizada de cada, com a apresentação das características e parâmetros fundamentais para o estudo, como os coeficientes de condutividade térmica.

\section{METODOLOGIA}

O procedimento inicialmente utilizado para o desenvolvimento desta pesquisa é o levantamento exploratório, com busca de dados a partir do servidor Proxy da UNIVATES, pesquisando em artigos científicos, dissertações e teses publicados nas bases Periódicos da CAPES (http://www.periodicos.capes.gov.br/), e na fonte internacional de pesquisa científica Science Direct (http://www.sciencedirect.com/), até o período de 2017. Denomina-se este levantamento de Metodologia Bibliométrica. Segundo Macias-Chapula (1988, citado por Vanti, 2002), a bibliometria é: 
[...] o estudo dos aspectos quantitativos da produção, disseminação e uso da informação registrada. A bibliometria desenvolve padrões e modelos matemáticos para medir esses processos, usando seus resultados para elaborar previsões e apoiar tomadas de decisões.

Para a pesquisa, foram utilizadas as palavras-chave: isolamento de edificações (building insulation), condutividade térmica (thermal conductivity), sustentabilidade (sustainability), conforto térmico (thermal comfort), materiais de construção (building materials), materiais naturais (natural materials), materiais não poluentes (non-polluting materials), eficiência energética (energy efficiency), consumo energético (energy consumption), isolamento térmico com fibras (fibrous thermal insulation) e desenvolvimento sustentável (sustainable development). Após a pesquisa, os resultados foram filtrados por área de interesse como: engenharia civil (civil engineering), arquitetura (architecture), conforto térmico (thermal comfort), isolamento térmico (thermal insulation), ambiente (environment) e condutividade térmica (thermal conductivity). Ao término da pesquisa com palavras chaves, analisou-se aproximadamente 40 pesquisas (artigos, dissertações e teses), que foram consideradas por apresentarem (prioritariamente) dados de condutividade térmica relacionados aos materiais naturais.

\section{RESULTADOS E DISCUSSÃO}

\section{Materiais naturais para isolamento térmico}

Uma boa gama de materiais naturais, e de composição de alguma sobra de produção agrícola vem sendo estudados em pesquisas, para avaliação das potencialidades de serem utilizados na construção civil, principalmente para isolamento térmico e conforto acústico. Segundo Asdrubali et al. (2015a),

$\mathrm{O}$ uso desses produtos não é generalizado e, em alguns casos, é limitado a um estágio experimental e laboratorial. [...] A sustentabilidade real dos materiais de isolamento considerados está ligada à sua disponibilidade; serem utilizados, preferencialmente, onde são colhidos, produzidos ou fabricados.

Junco

O junco pode ser considerado como o material de construção mais antigo utilizado para isolamento térmico, desde o período Neolítico para isolar edificações em períodos de frio. Em países do leste europeu são utilizados em telhados e paredes, tanto como isolamento interno ou externo (Figura 2), podendo ser revestidos com gesso (HISS REET, 2003, citado por ASDRUBALI et al., 2015a).

A condutividade térmica de um painel de junco pode ser considerada na faixa de 0,045 e 0,056 W/m.K, e sua densidade varia de 130 a $190 \mathrm{~kg} / \mathrm{m}^{3}$ (HISS REET, 2003, citado por ASDRUBALI et al., 2015a). No entanto, o valor de $0,045 \mathrm{~W} / \mathrm{m} . \mathrm{K}$ da condutividade térmica parece ser muito baixo. Pesquisas realizadas pelo método de placa quente, indicaram valores aproximados de $0,055 \mathrm{~W} / \mathrm{m} . \mathrm{K}$ para condutividade térmica (ASDRUBALI et al., 2012; ASDRUBALl et al., 2015b). 


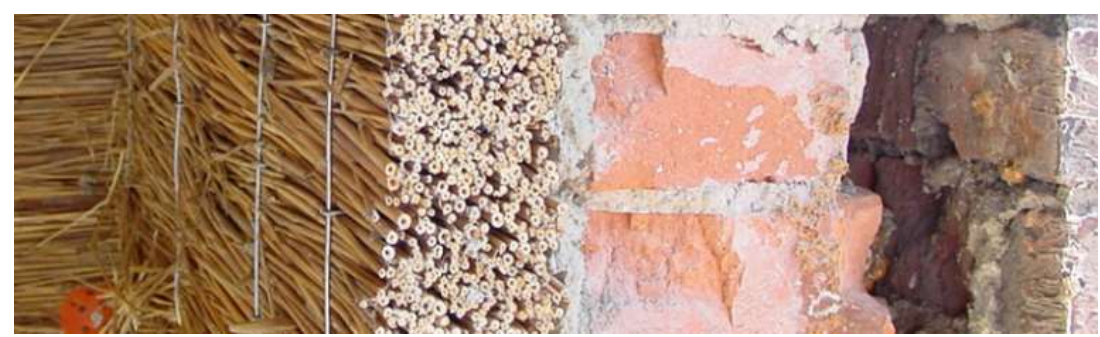

Figura 2: Painel de Junco aplicado em alvenaria de bloco cerâmico.

\section{Bagaço de Cana}

O Brasil é o principal produtor mundial de cana, responsável por $30,2 \%$ da área de plantio mundial, equivalente a 6,153 milhões de toneladas colhidas. Para cada tonelada de cana processada, é produzido aproximadamente $71 \mathrm{~kg}$ de açúcar e 42 litros de etanol, gerando como subproduto o bagaço. Atualmente, o bagaço vem sendo utilizado como biomassa para geração de energia. Novas pesquisar apontando para utilização na construção civil para fabricação de fibrocimento.

Ao estudar o efeito da densidade do bagaço na condutividade térmica, Manohar et al. (2006), analisaram amostras caracterizadas por densidade entre 70 e $120 \mathrm{~kg} / \mathrm{m}^{3}$. A amostra com melhor resultado apresentado foi com densidade de $100 \mathrm{~kg} / \mathrm{m}^{3}$ e uma condutividade térmica de $0,046 \mathrm{~W} / \mathrm{m} . \mathrm{K}$ (MANOHAR et al., 2006; MANOHAR, 2012).

Os experimentos desenvolvidos por Panyakaev et al. (2011), o valor da condutividade térmica ampliase para $0,049 \mathrm{~W} / \mathrm{m} . \mathrm{K}$ para o aglomerado com densidade de $250 \mathrm{~kg} / \mathrm{m}^{3}$, e atinge $0,055 \mathrm{~W} / \mathrm{m} . \mathrm{K}$ ao se testar a densidade de $350 \mathrm{~kg} / \mathrm{m}^{3}$. A pesquisa desenvolvida por Onésippe et al. (2010), em que o bagaço de cana foi adicionado a uma mistura de cimento com condutividade térmica de $0,62 \mathrm{~W} / \mathrm{m}$.K. Ao se adicionar $3 \%$ de fibras extraídas do bagaço, a condutividade térmica reduziu para 0,46 W/m.K (PANYAKAEV et al., 2011).

\section{Fibra de Folhas de Abacaxi}

Segundo Asdrubali et al. (2015a), a produção de abacaxi é geradora de resíduos, em que um deles é composto por suas folhas, que atualmente são utilizadas para geração de energia (térmica) ou simplesmente incineradas. Esses processos causam problemas ambientais, como poluição do ar e do solo, e podem ser reduzidos ao encontrar um uso inovador deste material no setor de construção (ASDRUBALI et al., 2015a).

No Brasil, a produção de abacaxi apresenta números consistentes, e estima-se que no ano de 2015 o plantio da fruta foi de 69.165 (ha), com um total de 1.801.415,00 unidades, tendo o estado do Pará como o maior produtor do país (EMBRAPA, 2016). Tangjuank (2011) estudou as propriedades de isolamento térmico desenvolvendo um painel composto de folhas de abacaxi trituradas e secas, com aplicação de látex de borracha natural. As amostras testadas apresentaram densidade entre 178 e $232 \mathrm{~kg} / \mathrm{m}^{3}$ e condutividade térmica entre 0,035 e 0,043 W/m.K. Observou-se um menor valor de condutividade para a amostra com uma densidade de $210 \mathrm{~kg} / \mathrm{m}^{3}$ (Tangjuank, 2011).

Em pesquisa desenvolvida por Kumfu et al. (2012), se investigou a condutividade térmica a partir de uma placa composta por fibras de abacaxi e borracha natural, com a utilização da técnica de prensagem a 
quente: neste caso, a amostra com densidade de $338 \mathrm{~kg} / \mathrm{m}^{3}$ apresentou uma condutividade térmica de 0,057 W/m.K (KUMFU et al., 2012, citado por ASDRUBALI et al., 2015).

\section{Casca de Arroz}

A produção mundial de arroz para a safra 2016/2017 está estimada em 481,5 milhões de toneladas (USDA), e dados da FAO apontam que o "consumo per capta mundial poderá chegar a 54,2 kg/pessoa na temporada 2016/17, maior que o da temporada anterior, de 54,1 kg/pessoa. O crescimento se deve ao aumento populacional na Ásia e à demanda otimista na África" (ALVES et al., 2017). No Brasil, a produção para período pode ter um crescimento de $8,5 \%$ em relação à safra anterior, colhendo 11,5 milhões de toneladas. O Rio Grande do Sul é o maior produtor do país, com previsão de colheita de 8,25 milhões de toneladas. Esta escala de produção gera uma grande quantidade de resíduos, que podem ser aplicados na composição de novos materiais (ALVES et al., 2017).

Em estudo desenvolvido por Yarbrough et al. (2005), foi avaliado o desempenho de isolamento térmico de painéis de aglomerados desenvolvidos com base na casca de arroz, apresentando condutividade térmica, a uma temperatura de $24^{\circ} \mathrm{C}$, entre 0,0464 e $0,0566 \mathrm{~W} / \mathrm{m} . \mathrm{K}$, em que o menor valor medido foi com densidade de $154 \mathrm{~kg} / \mathrm{m}^{3}$ (YARBROUGH et al., 2005, citado por ASDRUBALI et al., 2015a).

\section{Girassol}

No Brasil, a cultura de produção do Girassol está em desenvolvimento, principalmente para a produção no período da segunda safra (safrinha), podendo atingir uma produtividade média de $1.500 \mathrm{~kg} / \mathrm{ha}$, superior à média mundial. Desenvolvendo melhores condições de plantio, pode-se atingir uma produção aproximada com a da França, estimada em $2.500 \mathrm{~kg} / \mathrm{ha}$ (EMBRAPA, 2017).

Evon et al. (2014) produziram um material a base do composto residual de girassol durante os processos de refinaria, utilizando para produção de um painel de isolamento térmico. A amostra que apresentou melhor desempenho ficou com uma composição bastante frágil, e condutividade térmica superior ao ideal para ser utilizada como isolamento térmico (0,0885 W/m.K) (EVON et al., 2014). Em pesquisa desenvolvida por Binici et al. (2014), utilizou-se os talos de girassol para produção de dois materiais isolantes, adicionados a resíduos têxteis, em que a condutividade térmica mensurada foi, respectivamente, igual a $0,1642 \mathrm{~W} / \mathrm{m} . \mathrm{K}$ e $0,0728 \mathrm{~W} / \mathrm{m} . \mathrm{K}$ (BINICl et al., 2014).

\section{Palha}

A palha pode ser classificada como um subproduto do cultivo de cereais, e está disponível em grandes quantidades, a baixo custo, em um grande número de países. Tem sido um dos primeiros materiais a serem utilizados mundialmente em edificações sustentáveis. Normalmente, a palha utilizada para aplicação na construção de edificações é derivada do cultivo de trigo. (ASDRUBALI et al., 2015a). 
Em pesquisa desenvolvida por Goodhew et al. (2005), foi mensurada a condutividade térmica de $0,067 \mathrm{~W} / \mathrm{m} . \mathrm{K}$, para uma amostra desenvolvida com base de palha, e uma densidade de $60 \mathrm{~kg} / \mathrm{m}^{3}$ (GOODHEW et al., 2005). Pruteanu (2010) desenvolveu analises em dez amostras de palha, atingindo valores para condutividade térmica que variaram de $0,0515 \mathrm{~W} / \mathrm{m} . \mathrm{K}$ à $0,0740 \mathrm{~W} / \mathrm{m} . \mathrm{K}$, com média final de 0,061 W/m.K.

Marcos (2015) desenvolveu estudo a base de palha (Figura 3), com amostra apresentando densidade de $70 \mathrm{~kg} / \mathrm{m}^{3}$, a condutividade térmica registrada foi de $0,055 \mathrm{~W} / \mathrm{m} . \mathrm{K}$, similar aos estudos anteriores. No mesmo estudo, se estudou a capacidade de isolamento térmico utilizando Ramo de Cebola, que mesmo apresentando uma densidade distinta, a microestrutura fibrosa é muito similar a palha. A condutividade térmica aferida no teste é idêntica à da palha $(0,055 \mathrm{~W} / \mathrm{m} . \mathrm{K})$ (MARCOS, 2015).
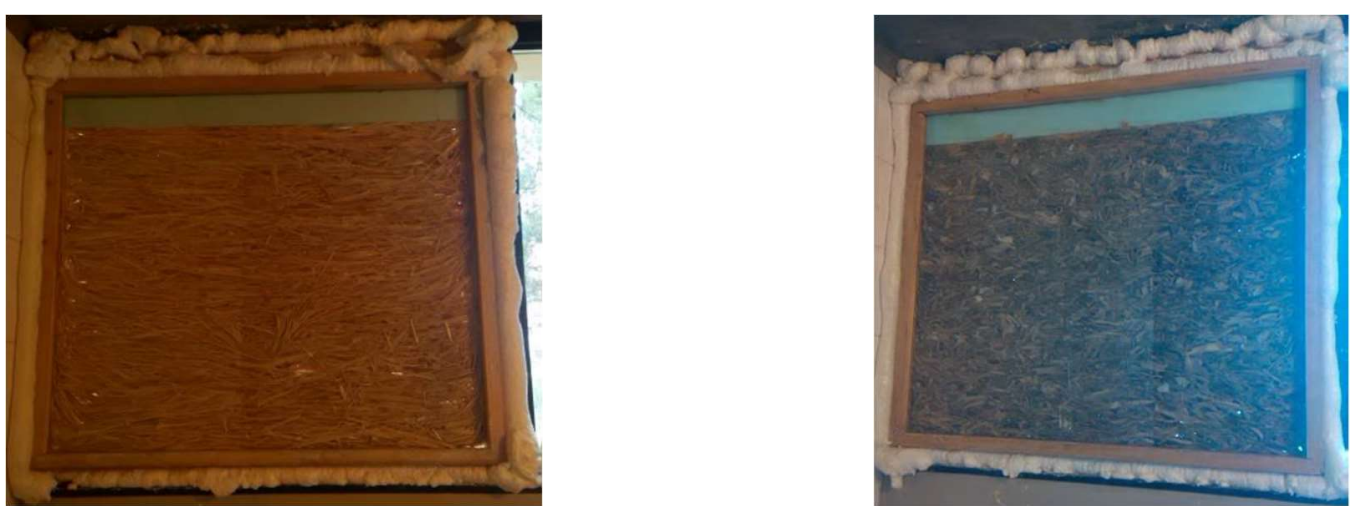

Figura 3: Palha e Ramo de Cebola. Fonte: Marcos (2015).

\section{Fibra da Palma de Óleo}

A Palma de Óleo (Elaeis guineensis) é a oleaginosa de maior importância mundial, e a produção mundial de óleo é estimada em 62,35 milhões de toneladas (safra 2014/2015). A palma de óleo se consolida com maior produção de óleo no mundo, com média de cinco toneladas por hectare, superando as demais oleaginosas cultivadas (BORGES et al., 2016).

Shinoj et al. (2011) apresenta trabalhos em que se utilizam os cachos da planta para produção de fibras (Figura 4) (SHINOJ et al., 2011). Manohar (2012) investigou o efeito da densidade na condutividade térmica da amostra de fibra de palma de óleo, e o menor valor de condutividade térmica foi de $0,055 \mathrm{~W} / \mathrm{m} . \mathrm{K}$, para uma densidade de $100 \mathrm{~kg} / \mathrm{m}^{3}$ (MANOHAR, 2012). Em estudo desenvolvido por Singh et al. (2003), a amostra pesquisada foi caracterizada por uma condutividade térmica de 0,293 W/m.K, alto para ser considerado como material isolante (SINGH et al., 2003). 


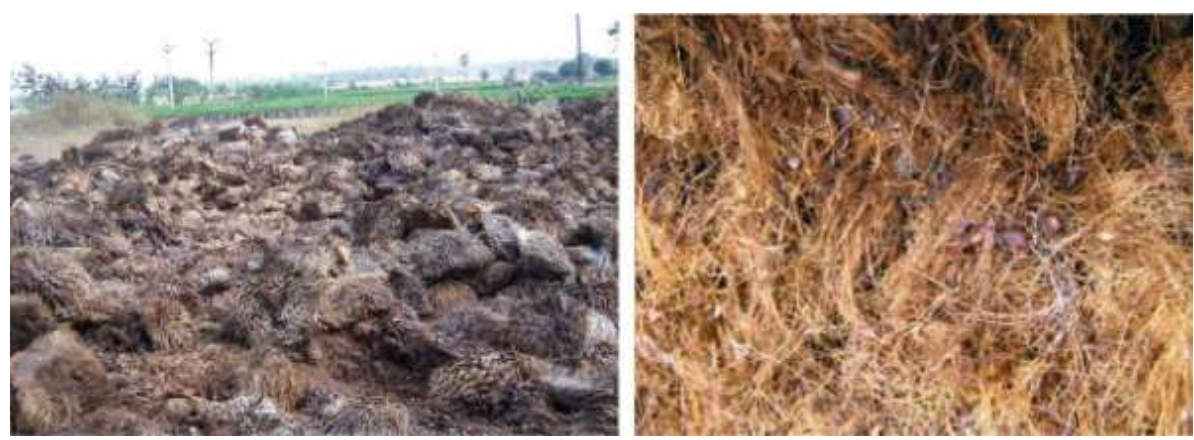

Figura 4: Fibra da Palma de Óleo. Fonte: Asdrubali et al. (2015).

\section{Casca de Pinheiro}

A casca de pinheiro pode se apresentar como uma alternativa viável para o desenvolvimento de material isolante. Segundo Marcos (2015), a casca de pinheiro da espécie pinheiros bravos (pinus pinaster Ainton), conforme Figura 5, apresentou uma densidade $250 \mathrm{~kg} / \mathrm{m}^{3}$, e condutividade térmica de $0,069 \mathrm{~W} / \mathrm{m} . \mathrm{K}$. A casca foi aplicada solta no preenchimento da placa do estudo, sem nenhum tipo de aglomerante (MARCOS, 2015).

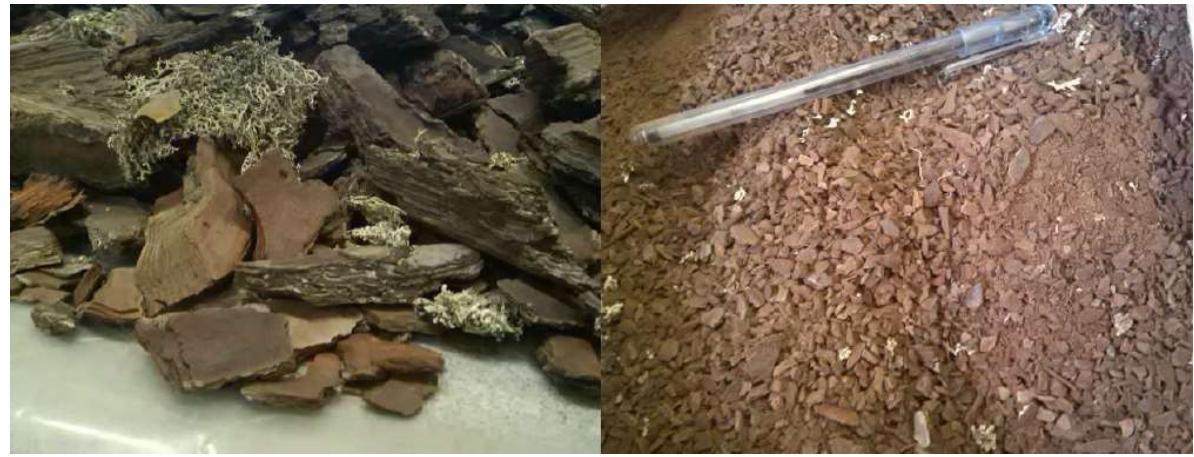

Figura 5: Casca de Pinheiro in natura e triturado. Fonte: Marcos (2015).

As florestas plantadas no Brasil se estendem por, aproximadamente, 7 milhões de hectares, em grande parte com plantação de pinus e eucalipto. A produção é destinada à indústria de papel, celulose, carvão vegetal, madeira serrada, produtos de madeira sólida e madeira processada, além da borracha. A retirada da casca no momento do corte do reflorestamento, pode ser uma alternativa viável no desenvolvimento do produto para isolamento térmico.

\section{Sabugo de Milho}

Segundo estimativas do Governo Brasileiro, a produção de milho para safra 2016/2017,

[...] deve alcançar 91,5 milhões de toneladas (37,5\% de crescimento), com 29,9 milhões de toneladas para a primeira safra e 61,6 milhões para a segunda. A área total do milho deve alcançar 17,1 milhões de hectares (ampliação de 7,3\%). No total, milho e soja representam quase $90 \%$ dos grãos produzidos no País.

Esta escala de produção, justificaria a utilização de sabugo de milho, que atualmente é tratado como resíduo, para utilização em isolamento térmico. Em dados apresentados por Pinto et al. (2011), algumas edificações de tabiques ${ }^{7}$ portuguesas foram executadas utilizando sabugo de milho misturado e terra como

\footnotetext{
${ }^{7}$ Técnica de construção difundida em Portugal entre os séculos XVIII e XIX (Pinto et al., 2011).
} 
materiais de preenchimento nas alvenarias, com intuito de reciclar esses resíduos agrícolas, sem o viés de melhorar as propriedades de isolamento térmico (PINTO et al., 2011).

Segundo Pinto et al. (2012), em uma placa aglomerada de sabugo de milho triturado com aplicação de cola de madeira, a condutividade térmica medida foi de $0,101 \mathrm{~W} / \mathrm{m} . \mathrm{K}$, ainda alto se comparado ao valor apresentado por Asdrubali et al. (2012) para ser um bom material de isolamento térmico (0,07 W/m.K) (PINTO et al., 2012; ASDRUBALI et al., 2012). Porém, em pesquisa desenvolvida por Marcos (2015), a placa desenvolvida sem a adição de cola de madeira apresentou o valor de 0,058 W/m.K para condutividade térmica, aferindo o material como viável para utilização, com densidade de $130 \mathrm{~kg} / \mathrm{m}^{3}$ (MARCOS, 2015).

\section{Fibra de Basalto}

O processo de fabricação da fibra de basalto (Figura 6) é mais simples que o processamento da fibra de vidro, possuindo uma composição menos complexa, em que o basalto fundido flui do forno através de uma bucha platino-ródio com furos, e as fibras podem ser retiradas da massa fundida sob pressão hidrostática (FIORE et al., 2015).

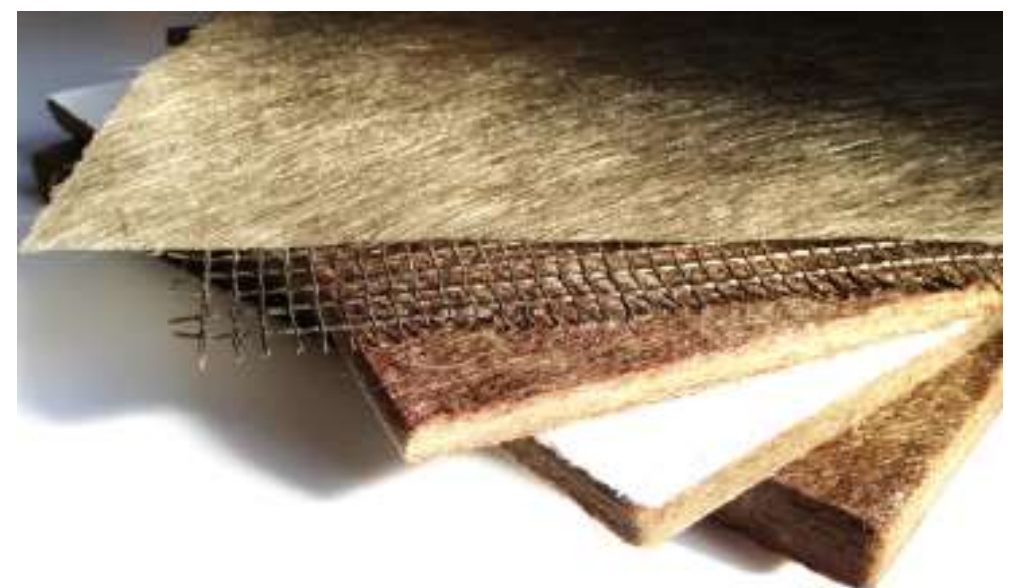

Figura 6: Painel isolante com base de fibra de basalto. Fonte: Moretti et al. (2016)

Destaca-se que o processo de fabricação da fibra de basalto é semelhante ao das fibras de vidro, mas com menor consumo de energia e sem aditivos. A rocha de basalto extraída é esmagada, e logo após é derretida em um forno aquecido a gás, com temperatura de $1450^{\circ} \mathrm{C}$. As fibras de basalto não têm nenhuma reação tóxica com o ar, água, ou outros produtos químicos, não são inflamáveis e à prova de explosão (MORETTI et al., 2016).

Os testes desenvolvidos apresentaram valores baixos de condutividade térmica. Para uma densidade de $165 \mathrm{~kg} / \mathrm{m}^{3}$, o valor de condutividade chega a 0,0312 W/m.K. Considerando uma densidade de $187 \mathrm{~kg} / \mathrm{m}^{3}$, a condutividade térmica é igual a 0,0320 W/m.K. Painéis com uma densidade inferior a $145 \mathrm{~kg} / \mathrm{m}^{3}$ não foram considerados nos testes devido à sua baixa resistência mecânica (MORETTI et al., 2016). Os autores pesquisados salientam que a fibra de basalto é uma substituição importante para o amianto (atualmente aplicado em partes da construção civil), e é adequado para aplicações que requerem resistência contra altas temperaturas, a ácidos e solventes, com durabilidade, resistência mecânica e baixa absorção de água. Por 
serem resistentes, podem ser aplicadas como elemento de proteção contra incêndio (FIORE et al., 2015; MORETTI et al., 2016).

\section{Painel de Isolamento a Vácuo (VIP)}

Relacionado a materiais inovadores, estudo desenvolvido na China aprofunda a análise sobre componentes para isolamento térmico, composto de materiais com fibra de vidro e de alumínio laminado (Vacuum Insulation Panel - VIP), com índice de condutividade térmica de 0,030 W/m.K (BOAFO et al., 2014). Em pesquisa realizada na Coréia do Sul, destaca-se a eficiência do VIP, porém, ressalta que os elementos utilizados como suporte para a instalação do material são causadores de pontes térmicas, ocasionando transferência de calor (BOAFO et al., 2015).

Mujeebu et al. (2016) desenvolveu estudo baseado em simulações computacionais com aplicação de diferentes materiais de construção. Em duas simulações propõe a utilização de VIP, em que se destaca a baixa condutividade térmica, com um índice de 0,003 W/m.k (MUJEEBU et al., 2016). Dados fornecidos pela fabricante de diversos modelos de VIP, a va-Q-tec (2016) descreve nas especificações técnicas dados relacionados a condutividade térmica, uma variação de $0,0028 \mathrm{~W} / \mathrm{m} \cdot \mathrm{k}$ à $0,009 \mathrm{~W} / \mathrm{m} \cdot \mathrm{k}$, variando conforme a densidade e espessura da placa definida para aplicação (Figura 7) (VA-Q-TEC, 2016).

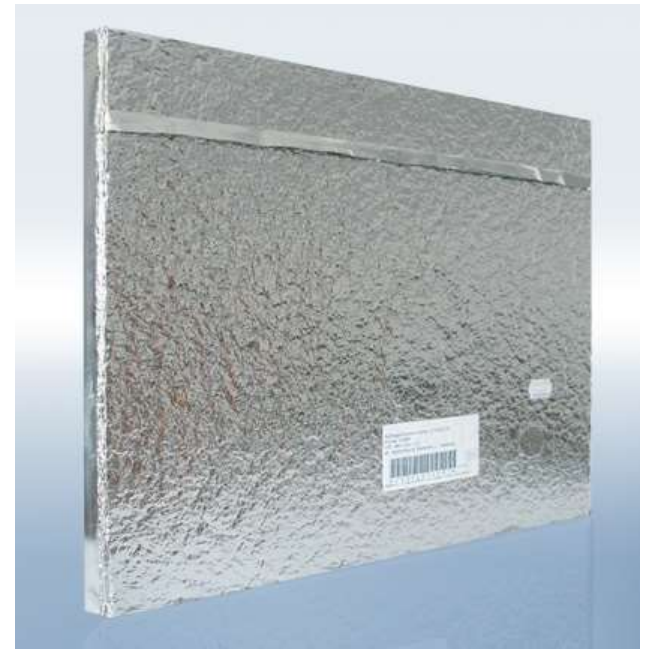

Figura 7: Painel de Isolamento a Vácuo (VIP - Modelo va-Q-vip). Fonte: va-Q-tec (2016).

\section{CONCLUSÕES}

Com a crescente preocupação ambiental, destaca-se a importância da utilização de componentes construtivos que tenham menor consumo energético e de combustíveis fósseis no processo de fabricação, conforme destacado por Dutra (2010). Pode-se verificar no Gráfico 1, que para os materiais naturais analisados, grande parte apresentou um índice de condutividade térmica ideal para serem utilizados como isolamento térmico.

Assim, tornam-se relevantes os experimentos desenvolvidos com materiais naturais analisados neste estudo, sendo alternativas viáveis na substituição aos elementos fabricados de forma convencional. Os dados de condutividade térmica pesquisados serão fundamentais na utilização de simulação em projetos de 
edificações, e na avaliação de desempenho energético. Porém, como os testes são desenvolvidos em laboratórios para determinação da condutividade térmica, surge a importância de serem aplicado e analisados em protótipos com escala real, aplicados juntamente a materiais de construção convencionais, para assim, avaliar a sua durabilidade, resistência, e seu comportamento como material de isolamento térmico, e principalmente, estudar o período de tempo em que o material mantem as propriedades isolantes.

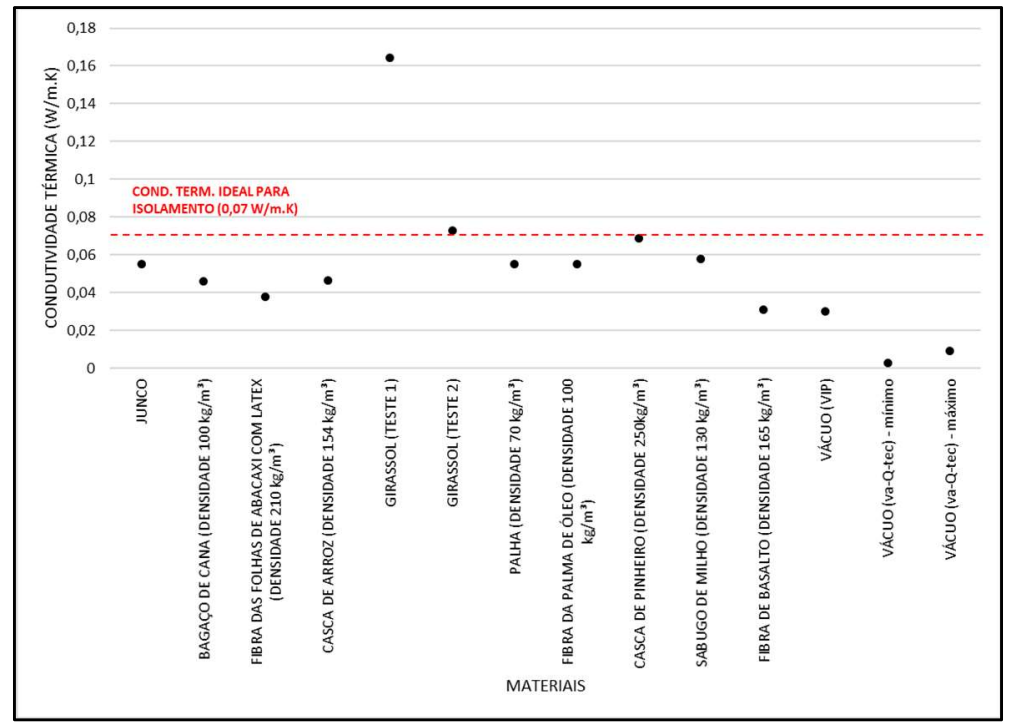

Gráfico 1: Comparativo de condutividade térmica para materiais naturais.

Importante destacar que para grande parte dos elementos pesquisados, foi analisado o comportamento acústico dos materiais, com desempenho satisfatório ao serem comparados com materiais convencionais. Para estudos futuros, pode-se pesquisar informações de resistência ao fogo (incombustibilidade), fundamental para segurança em projeto de edificações, complementando com informações de Avaliação do Ciclo de Vida $\left(\mathrm{ACV}^{8}\right)$.

Em relação a incombustibilidade, é aconselhavel que se siga o método da norma ISO 1182 - Reaction to fire tests for products - Non combustibility test (Testes de reação ao fogo para produtos - Teste de não combustão). Este método determina que, mesmo os materiais não sendo totalmente inertes, produzam uma pequena quantidade de calor e chama ao serem expostos a uma temperatura aproximada de 750 으 (ISO, 2010). Com a realização deste teste, é possivel verifiacar a segurança de materiais de construção, quanto a sua capacidade de propagação de incêndios em edificações.

Os dados relacionados ao ACV de elementos pesquisados, segundo Patzlaff et al. (2010), ao se analizar o aspecto ambiental na construção civil, "a sustentabilidade pode ser abordada com foco nos impactos gerados pelos materiais e sistemas construtivos", podendo-se utilizar como ferramenta a ACV, por estar [...] "alinhada aos assuntos discutidos na Conferência Rio+10, em termos de quais ações devem ser planejadas para o desenvolvimento de políticas de produção e consumo numa forma de melhorar produtos e serviços oferecidos, reduzindo os impactos ambientais" [...], auxiliar na identificação de oportunidades para

\footnotetext{
${ }^{8}$ A ACV estuda os aspectos ambientais e os impactos potenciais ao longo da vida de um produto (isto é, do "berço ao túmulo"), desde a aquisição da matéria-prima, passando por produção, uso e disposição. As categorias gerais de impactos ambientais que necessitam ser consideradas incluem o uso de recursos, a saúde humana e as consequências ecológicas" (ABNT, 2001).
} 
melhoria dos "aspectos ambientais de produtos em vários pontos de seu ciclo de vida" (da produção ao uso final); a selecionar os indicadores adequados para mensurar o desempenho ambiental de um produto/processo; e aspectos de divulgação de um produto (PATZLAFF et al., 2010). Salienta-se que "a ACV não aborda os aspectos econômicos ou sociais de um produto", porém deve ser levado em conta em análises futuras, para se verificar a viabilidade de fabricação de novos produtos (ABNT, 2001).

\section{REFERÊNCIAS}

ALVES, L.; BRAGHETTA, M. A.; SBRAVATTI, A.. Arroz/CEPEA: Colheita deve pressionar valor no 10 semestre, mas estoque pode sustentar no 2‥ Piracicaba: CEPEA, 2017.

ASDRUBALI, F.; SCHIAVONI, S.; HOROSHENKOV, K. V.. Review of sustainable materials for acoustic applications. Building Acoustics, Londres, v.19, n.4, p.283-312, 2012.

ASDRUBALI, F.; D’ALESSANDRO, F.; SCHIAVONI, S.. A review of unconventional sustainable building insulation materials. Sustainable Materials and Technologies, v.4, p.1-17, 2015a.

ASDRUBALI, F.; D'ALESSANDRO, F.; SCHIAVONI, S.; MENCARELLI, N.. Sound absorption properties of reed. In: INTERNATIONAL CONGRESS ON SOUND AND VIBRATION, 22. Anais. Florença, 2015b.

ABNT. Associação Brasileira de Normas Técnicas. NBR ISO 14040: Gestão ambiental: Avaliação do ciclo de vida: Princípios e estrutura. Rio de Janeiro, 2001.

ABNT. Associação Brasileira de Normas Técnicas. NBR 15220-1: Desempenho Térmico de Edificações - Parte 1: Definições, símbolos e unidades. Rio de Janeiro, 2005.

ABNT. Associação Brasileira de Normas Técnicas. NBR 15220-2: Desempenho Térmico de Edificações - Parte 2: Métodos de cálculo da transmitância térmica, da capacidade térmica, do atraso térmico e do fator solar de elementos e componentes de edificações. Rio de Janeiro, 2005.

ABNT. Associação Brasileira de Normas Técnicas. NBR 15220-4: Desempenho Térmico de Edificações - Parte 4: Medição da resistência térmica e da condutividade térmica pelo princípio da placa quente protegida. Rio de Janeiro, 2005.

ABNT. Associação Brasileira de Normas Técnicas. NBR 15575: Edifícios habitacionais - Desempenho. Rio de Janeiro, 2013.

BINICI, H.; EKEN, M.; DOLAZ, M.; AKSOGAN, O.; KARA, M.. An environmentally friendly thermal insulation material from sunflower stalk, textile waste and stubble fibres.

Construction and Building Materials, v.51, n.31, p.24-33, 2014.

BOAFO, F. E.; CHEN, Z.; LI, C.; LI, B.; XU, T.. Structure of vacuum insulation panel in building system. Energy and Buildings, v.85, p.44-653, 2014.

BOAFO, F. E.; KIMA, J.T.; CHEN, Z.. Configured cavity-core matrix for vacuum insulation panel: Concept, preparation and thermophysical properties. Energy and Buildings, v.97, n.15, p.98-106, 2015.
BORGES, A. J.; COLLICCHIO, E.; CAMPOS, G. A.. A cultura da palma de óleo (Elaeis guineenses Jacq.) no Brasil e no mundo: aspectos agronômicos e tecnológicos - uma revisão. Revista Liberato, Novo Hamburgo, v.17, n.27, p.01-118, 2016.

CAMBEIRO, F. P.; ARMESTO, J.; BARBEITO, F. P.; BASTOS, G.. Perspectives on Near ZEB Renovation Projects for Residential Buildings: The Spanish Case. Energies, v.9, n.8, p.2-16, 2016.

DUTRA, M. R.. Caracterização de revestimentos em fachadas ventiladas: Análise do comportamento. Dissertação (Mestrado em Engenharia Civil) - Universidade Técnica de Lisboa, Porto, 2010.

EICHHAMMER, W.; FLEITER, T.; SCHLOMANN, B.; FABERI, S.; FIORETTO, M.; PICCIONI, N.; LECHTENBÖHMER, S.; SCHÜRING, A.; RESCH, G.. Study on the Energy Savings Potentials in EU Member States, Candidate Countries and EEA Countries. Vienna, 2009.

EMBRAPA. Abacaxi. Brasília: EMBRAPA, 2016.

EMBRAPA. Girassol. Brasília: EMBRAPA, 2017.

EPE. Empresa de Pesquisa Energética. Consumo mensal de energia elétrica por classe (regiões e subsistemas) - 20042017. Brasília: Ministério de Minas e Energia, 2017.

EPE. Empresa de Pesquisa Energética. O Compromisso do Brasil no Combate às Mudanças Climáticas: Produção e Uso de Energia. Brasília: Ministério de Minas e Energia, 2016.

EVON, P.; VANDENBOSSCHE, V.; PONTALIER, P. Y.; RIGAL, L. New thermal insulation fiberboards from cake generated during biorefinery of sunflower whole plant in a twin-screw extruder. Industrial Crops and Products, v.52, p.354-362, 2014.

FIORE, V.; SCALICI, T.; DI BELLA, G.; VALENZA A.. A review on basalt fibre and its composites. Composites Part B, v.74, n.1, p.74-94, 2015.

GOODHEW, S.; GRIFFITHS, R.. Sustainable earth walls to meet the building regulations. Energy and Buildings, v.37, n.5, p.451-459, 2005.

IOS. International Organization for Standardization. Building materials, non-combustibility test - ISO 1182. Switzerland, 2010.

JOURDA, F. H.. Pequeno Manual do Projeto Sustentável. Barcelona: Gustavo Gili, 2012. 
MCNAUGHT, A. D.. Compendio de terminologia química. Madrid: Sintesis, 2005

MANOHAR, K.; RAMLAKHAN, D.; KOCHHAR, G.; HALDAR, S. Biodegradable fibrous thermal insulation. J. Braz. Soc. Mech. Sci. \& Eng., Rio de Janeiro, v.28, n.1, p.45-47, 2006.

MANOHAR, K.. Experimental Investigation of Building Thermal Insulation from Agricultural By-products. British Journal of Applied Science \&

Technology, Londres, v.2, n.3, p.227-239, 2012.

MARCOS, J. S. L.. Materiais Naturais Alternativos de Isolamento Térmico. Dissertação (Mestrado em Engenharia Civil) - Universidade de Trás-os-Montes e Alto Douro, Vila Real, 2015.

MARQUES, B. R.; MARTINS, L. J. R.; ANDRADE, F. A. Poliuretano derivado de óleo de mamona: de meio ambiente a biocopatibilidade. In. ENCONTRO CIENTÍFICO UNISALESIANO, 2. Anais. Lins: UNISALESIANO, 2009.

MORETTI, E.; BELLONI, E.; AGOSTI, F.. Innovative mineral fiber insulation panels for buildings: Thermal and acoustic characterization. Applied Energy, v.169, n.1, p.421-432, 2016.

MUNIZ, F. G. L.; CARACRISTI, I.. Urbanização, Conforto Térmico e Análise Sazonal Microclimática da Cidade de Sobral/CE. Revista da Casa da Geografia de Sobral, Sobral, v.17, n.1, p.4-17, 2015.

ONÉSIPPE, C.; PASSE-COUTRIN, N.; TORO, F.; DELVASTO, S.; BILBA, K.; ARSÈNE, M. A.. Sugar cane bagasse fibres reinforced cement composites: Thermal considerations. Composites: Part A, v.41, n.4, p.549-556, 2010.

PANYAKAEV, S.; FOTIOS, S.. New thermal insulation boards made from coconut husk and bagasse. Energy and Buildings, v.43, n.7, p.1732-1739, 2011.

PATZLAFF, J. O.; KERN, A. P.; GONZÁLEZ, M. A. S.. Projeto de Edificações com Apelo Sustentável: Elementos para $A$ Construção de um Sistema de Apoio à Decisão. Revista Produção Online, v.10, n.3, p.479-503, 2010.

PRUTEANU, M.. Investigations Regarding the Thermal Conductivity of Straw. Buletinul Institutului Politehnic Din Lasi. Sectia Constructii, Arhitectura, v.56, n.3, p.9-16. Geórgia, 2010.
PINTO, J.; PAIVA, A.; VARUM, H.; COSTA, A.; CRUZ, D; PEREIRA, S.; FERNANDES, L.; TAVARES, P.; AGARWAL, J.. Corn's cob as a potential ecological thermal insulation material. Energy and Buildings, v.43, n.8, p.1985-1990, 2011.

PINTO, J.; CRUZ, D.; PAIVA, A.; PEREIRA, S.; TAVARES, P.; FERNANDES, L.; VARUM, H.. Characterization of corn cob as a possible raw building material. Construction and Building Materials, v.34, p.28-33, 2012.

PROCEL. Resultados PROCEL 2016: Ano Base 2015. Brasília: Eletrobrás, 2016.

PROCEL INFO. Edificação. Brasília: Eletrobrás, 2016.

SINGH, K.; SAXENA, N. S.; SREEKALA, M. S.; THOMAS, S.. Temperature dependence of the thermal conductivity and thermal diffusivity of treated oil-palm-fiber-reinforced phenolformaldehyde composites. Journal of Applied Polymer Science, v.89, n.13, p.3458-3463, 2013.

SHINOJ, S.; VISVANATHANB, R.; PANIGRAHI, S.; KOCHUBABUA, M.. Oil palm fiber (OPF) and its composites: A review. Industrial Crops and Products, v.33, n.1, p.7-22, 2011.

SPINELLI, R.; ALVES, A.; KONRAD, O.. Cidade e Edificações: A busca por um novo modelo. Destaques Acadêmicos, Lajeado, v.5, n.4, p.173-186, 2013.

SPINELLI, R.; KONRAD, O.; CAMBEIRO, F. P.; AHLERT, E.; SPINELLI, F. B.; QUADROS, E. A.. Bioclimatic Strategies for The City of Lajeado/Rs-Brazil, Using Data Analysis of Regional Climate. Revista Brasileira de Climatologia, Curitiba, v.21, n.2, p. 53-171, 2017.

TANGJUANK, S.. Thermal insulation and physical properties of particleboards from pineapple leaves. International Journal of Physical Sciences, v.6, n.19, p.4528-4532, 2011.

VA-Q-TEC. Painel de Isolamento a Vácuo: Vacuum Insulation Panel. Würzburg, 2016.

VANTI, N. A. P.. Da bibliometria à webometria: uma exploração conceitual dos mecanismos utilizados para medir o registro de informação e a difusão do conhecimento. Revista Ciência da Informação. Brasília, 2002.

WIEBECK, H.; HARADA, J.. Plásticos de Engenharia: Tecnologia e Aplicações. São Paulo: Artliber, 2005.

A CBPC - Companhia Brasileira de Produção Científica (CNPJ: 11.221.422/0001-03) detém os direitos materiais desta publicação. Os direitos referem-se à publicação do trabalho em qualquer parte do mundo, incluindo os direitos às renovações, expansões e disseminações da contribuição, bem como outros direitos subsidiários. Todos os trabalhos publicados eletronicamente poderão posteriormente ser publicados em coletâneas impressas sob coordenação da Sustenere Publishing, da Companhia Brasileira de Produção Científica e seus parceiros autorizados. Os (as) autores (as) preservam os direitos autorais, mas não têm permissão para a publicação da contribuição em outro meio, impresso ou digital, em português ou em tradução. 